PS2 - 197

doi:10.1017/cjn.2016.371

\section{DICER Governs Characteristics of Glioma Stem Cells and the Resulting Tumors in Xenograft Mouse Models of GBM}

S. Mansouri ${ }^{1}$, S. Singh, A. Alamsahebpour, K. Burrell, M. Li, M. Karabork, C. Ekinci, I. Solaroglu, J.T. Chang, K. Aldape, G. Zadeh ${ }^{I}$ University of Toronto,Toronto, ON mansourisheila@gmail.com

The RNAse III endonuclease DICER is a key regulator of microRNA (miRNA) biogenesis and is frequently down-regulated in a variety of malignancies. We characterized the role of Dicer in glioblastoma (GB), specifically demonstrating its effects on the ability of glioma stem-like cells to form tumors in a mouse model of GB. DICER silencing in glioma stem-like cells (GSCs) reduced their stem cell characteristics, while tumors arising from these cells were more aggressive, larger in volume, and displayed a higher proliferation index and lineage differentiation. The resulting tumors, however, were more sensitive to radiation treatment. Our results demonstrate that DICER affects the tumorigenic potential of GSCs, providing a platform for analysis of specific relevant miRNAs and development of potentially novel therapies against GB.

PS2 - 201

doi:10.1017/cjn.2016.372

\section{Bone Marrow-Derived Mesenchymal Stem Cells-Mediated} Radioresistance in Glioma

\section{T. Zhao ${ }^{l}$, G. Zadeh \\ ${ }^{1}$ University of Toronto, Toronto, $O N$ tansy.zhao@mail.utoronto.ca}

Ionizing radiation (IR) is one of the conventional post-surgical treatments for Glioblastoma Multiforme (GBM). Mesenchymal stem cells (MSCs) constitute a subpopulation of bone marrow derived cells which are actively recruited to the site of radiation and/or tumour microenvironment (TME), both of which have important implications for neovascularization and tumor progression. The goal of this project is to investigate the functional contribution of MSCs in the TME. We postulate that Bone Marrow-MSCs promote radio-resistance in GBM via cell cycle arrest. We tested the effect of MSC on U87 glioblastoma cell line in response to IR. We found that MSC co-culture, MSCconditioned media (MSCCM) and irradiated MSC-conditioned media (MSCIRCM) did not reduce IR-induced p53 (ser15) phosphorylation, signifying intact p53-dependent DNA damage pathway in all conditions. However, both MSCCM and MSCIRCM temporally increased phospho-Chk2, a kinase involved in ATM-dependent cascade and cell cycle arrest. This increase occurred at 24 hours and reverted to baseline levels by 48 hours. Interestingly, IR (15Gy) caused transiently heightened metabolic rate under MSC and MSC IRCM as opposed to IR-null treatment at 48 hours elevated cell proliferation. MSCCM, but not MSCIRCM, marginally reduced caspase 3/7-dependent apoptotic levels. The combination of IR and MSCCM as well as MSCIRCM first increased protein level of phospho-Chk2 at 24 hours; followed by increased metabolic rate at 48 hours; and lastly, boosted proliferation at 72 hours. This data combined proposes plausible machinery for BM-MSC mediated radio-resistance by initiating cell cycle arrest in tumour cells for DNA damage repair.
PS2 - 203

doi:10.1017/cjn.2016.373

Bone Marrow-Derived Mesenchymal Stem Cells Reverse Ionizing Radiation-Induced Active State of Endothelial Cells in Glioblastoma

T. Zhao ${ }^{l}$, J. Metcalf, G. Zadeh

${ }^{1}$ University of Toronto, MacFeeters-Hamilton Brain Tumor Centre tansy.zhao@mail.utoronto.ca

As the central component of heightened vascularization in glioblastoma (GBM), endothelial cells (EC) are arguably the most responsive stromal cells in the tumour microenvironment during conventional treatment using ionizing radiation (IR). Although the inherent tumor tropism and angiogenic property of mesenchymal stem cells (MSC) has been documented in many cancer types including GBM, little is known about the function of MSC that are recruited to the GBM tumor microenvironment. The purpose of this study was to elucidate the effect of MSC on irradiated EC. Here we studied the influence of IR, MSC or MSC condition media (CM) on human umbilical vein endothelial cells (HUVECs). IR was found to up-regulate mRNA levels of CXCL5, CXCL10, ICAM1, VCAM1, VEGF-c and tissue factor (TF) in a dosedependent manner whereas MSC co-culture boosted the expression of ICAM1, VCAM1, VEGF-c, TF, and MCP3. An additive effect of IR and MSC-culture was most detected with respect to ICAM1, TF and CXCL5 under 2Gy. MSC co-culture decreased IR-induced phospho-p53 in HUVECs at 15Gy. In addition, IR decreased the level of phospho-Akt in HUVECs as well as cell proliferation. Notably, both effects were countered by MSC CM. Interestingly, up-regulation of the same set of IR-driven genes in GBM was positively correlated with poor survival in the TCGA GBM database, a correlation that was lost if using gene list that was obtained from IR and MSC combine. These findings suggest that MSC promotes angiogenesis in GBM by overturning the IRinduced active state of endothelial cells and rendering them radioresistant.

PS2 - 209

doi:10.1017/cjn.2016.374

RXFP1 Promotes Temozolomide Chemoresistance by STAT3 Signaling Pathway Activation

T. Thanasupawat ${ }^{l}$, A. Glogowska ${ }^{l}$, M. Burg ${ }^{l}, J . K_{r c e k}{ }^{l, 2}, J . B e i k o^{2}$, M. Pitz ${ }^{3,4}$, S. Hombach-Klonisch ${ }^{1,5}$, T. Klonisch ${ }^{1,2,6}$

${ }^{I}$ Department of Human Anatomy and Cell Science, Winnipeg, MA

${ }^{2}$ Department of Surgery, University of Manitoba, Winnipeg, MA

${ }^{3}$ Department of Internal Medicine, University of Manitoba

${ }^{4}$ Department of CancerCare Manitoba, Winnipeg, MA

${ }^{5}$ Department of Obstetrics, Gynecology and Reproductive

Sciences, University of Manitoba, Winnipeg, MA

${ }^{6}$ Department of Medical Microbiology and Infectious Diseases University of Manitoba, Winnipeg, MA

We identified the $G$ protein coupled relaxin receptor RXFP1 in glioblastoma (GB) and discovered Clq-tumor necrosis factor related protein 8 (CTRP8) as a novel ligand of RXFP1 in this common and most aggressive form of brain tumor. The known RXFP1 ligand relaxin isoform 2 (RLN2) is not expressed in the 
brain indicating that RXFP1 signaling in GB is activated by auto/paracrine secretion of CTRP8. In GB, RXFP1 signaling included $\mathrm{PI} 3 \mathrm{~K}$ and $\mathrm{PKC}$ activation and resulted in the increased production and secretion of lysosomal protease cathepsin B, a known prognostic marker of GB. In the present study, we have investigated the potential role of RXFP1 in chemoresistance to the commonly used DNA alkylating drug temozolomide (TMZ) in human GB. Upon TMZ treatment, CTRP8/RLN2 mediated activation of RXFP1 was able to mitigate DNA damage in human primary GB cells and enhanced their survival. Activation of RXFP1 resulted in STAT3 pathway activation and the RXFP1- and STAT3-dependent up-regulation of proteins and their activity deemed important for TMZ-induced DNA damage repair. Furthermore, RXFP1 activation resulted in the up-regulation of key anti-apoptotic factors in human GB cells. Our results indicate a novel role for the CTRP8-RXFP1 ligand-receptor system in STAT3-dependent cell invasion, TMZ chemoresistance, and survival and identify RXFP1 as a new protective $\mathrm{G}$ protein coupled receptor in GB cells.

PS2 - 217

doi:10.1017/cjn.2016.375

\section{Treatment of Glioblastoma Brain Tumor-Initiating Cells with} Microglial and Macrophage-Derived Cytokines

\author{
C.C. Poon ${ }^{\text {I }, ~ S . ~ S a r k a r, ~ M . L . ~ H u g h e s, ~ V . W . ~ Y o n g, ~ J . J . ~ K e l l y ~}$ \\ ${ }^{I}$ University of Calgary, Calgary, $A B$ \\ candicecpoon@gmail.com
}

The most common brain cancer, glioblastoma (GBM), also has the worst five-year survival rates of all cancers. This is due in part to cancer stem cells, known as brain tumor initiating cells (BTICs), which initiate GBM and are resistant to current therapies. Thus, it is important to study therapeutics targeting BTICs. Innate immune cells, microglia and macrophages (MMs), are also important GBM-associated cells. GBM influences MMs to promote tumor growth. However, my research group discovered that an old drug, amphotericin B, re-stimulates MMs to secrete cytokines that inhibit BTIC self-renewal (Nature Neurosci 17:46-55, 2014). Hence, I hypothesize these cytokines are potential GBM treatments. Methods: Twenty genetically diverse BTIC lines were exposed to interleukin-8 (IL8), monocyte chemoattractant protein-1 (MCP1), and tumor necrosis factor-alpha (TNF), the cytokines secreted most abundantly by amphotericin-stimulated MMs. Assays for proliferation, differentiation, apoptosis, and cell cycle arrest were performed. BTICs were then co-cultured with human GBMderived MMs stimulated with TNF. Lastly, the relationship between TNF receptors (TNFR1/2) and presumed BTICs was determined in frozen human GBM sections by immunofluorescence. Results: TNF had the most potent inhibitory effect on BTIC proliferation. Also, TNF was able to increase BTIC differentiation, and induce apoptosis and G1 cell cycle arrest. In MM-BTIC co-culture, TNF stimulated MMs to decrease sphere formation. Interestingly, in human GBM tissue TNFR1 colabeled with OLIG2, a major transcription factor expressed in presumed BTICs, implicating TNF as a BTIC-specific treatment. Conclusion: TNF is a promising candidate for the treatment of GBM.

\section{PATHOLOGY}

PS2 - 195

doi:10.1017/cjn.2016.376

\section{Community \\ R. Nejad ${ }^{1}$, D.N. Louis, K. Aldape, G. Zadeh \\ ${ }^{1}$ University of Toronto, Toronto, ON \\ romina.nejad@mail.utoronto.ca}

Integrating Molecular Workings into the World Health Organization (WHO) Classification of Tumours of the Central Nervous System: A Survey from the Neuro-Oncology

Tumours of the central nervous system are currently classified based on the 2007 WHO Classification of Tumours, which uses histological features to classify and grade these heterogeneous tumors. With recent advances in the development of clinically relevant molecular signatures, there is an interest to incorporate appropriate molecular markers in to the classification. The views of the neuro-oncology community on such changes would be informative for advising this process. METHODS A survey with 8 questions regarding molecular markers in tumor classification was sent to an email list of Society of Neuro-Oncology members and attendees of prior meetings $(n=5065)$. There were 403 respondents. Analysis was performed using whole group response and based on self-reported sub-specialty. RESULTS Survey results among all respondents show support for incorporating our molecular knowledge of brain tumors into the WHO classification (>80\%). As one example, $96 \%$ of respondents responded that the integration of $1 \mathrm{p} / 19 \mathrm{q}$ co-deletion into the molecular classification of oligodendroglioma was "very" or "critically" important for the management of grade III gliomas. While 30\% of all respondents believe that IDH mutation status should affect overall management of GBM. Interestingly, there was some variability among subspecialties for certain aspects, and as one example neuropathologists were slightly more inclined to disagree that molecular markers should be included in the WHO classification (25\% for neuropathologists versus $13 \%$ overall). CONCLUSION Based on a survey provided to the neuro-oncology community, we report strong support for the integration of molecular markers into the WHO classification of brain tumors, as well as for using an integrated "layered" diagnostic format.

PS2 - 205

doi:10.1017/cjn.2016.377

\section{Application of Computer-Assisted Diagnostics for Immunohistochemistry Analysis of Gliomas}

\section{A. Alamsahebpour ${ }^{l}$, G. Zadeh, K. Aldape \\ ${ }^{1}$ University of Toronto, Toronto, ON aalamsah@uhnresearch.ca}

In the current practice of pathology, the identification of cell markers and their respective distribution represents an indispensable dialogue for diagnostic, predictive, therapeutic, and research purposes. Early immunohistochemical protocols were limited to direct, fluorescent labeled antibodies, yielding quick results but lacking sensitivity. More recently, the use of indirect techniques -utilization of enzyme labels - and various detection systems have continued to advance the complexity of IHC, 